\title{
Quality of life of university students during the COVID-19 pandemic: Assessing the level of quality of life and the associated factors after the end of movement lockdown
}

\section{Mohammad Farris Iman Leong Bin Abdullah ( $\sim$ farris@usm.my )}

Advanced Medical and Dental Institute, Universiti Sains Malaysia https://orcid.org/0000-0002-77624052

Nor Shuhada Murad

Advanced Medical and Dental Institute, Universiti Sains Malaysia

Soo Huat Teoh

Advanced Medical and Dental Institute, Universiti Sains Malaysia

Mohd Afifuddin Mohamad

Advanced Medical and Dental Institute, Universiti Sains Malaysia

\section{Research Article}

Keywords: Quality of life, depression, anxiety, social support, COVID-19 pandemic, university students

Posted Date: December 28th, 2020

DOI: https://doi.org/10.21203/rs.3.rs-104496/v2

License: (c) (1) This work is licensed under a Creative Commons Attribution 4.0 International License.

Read Full License 


\section{Abstract}

Purpose: Despite the significant impact of COVID-19 on mental health among university students, data on the related quality of life (QoL) are lacking in this group of the population. This study aimed to evaluate quality of life (QoL) and determine its association with various factors and social support among university students during the COVID-19 pandemic after the end of movement lockdown.

Methods: This was an online, cross-sectional study recruited a total of 316 participants. Participants were administered a self-reported questionnaire to gather data on demographic, personal, clinical and psychological characteristics; the 21-item depression, anxiety and stress scale (DASS-21) to assess the severity of their depressive, anxiety and stress symptoms; the multidimensional scale of perceived social support (MSPSS) to assess the degree of social support; and the World Health Organization quality of life-BREF (WHOQoL-BREF) to assess QoL.

Results: The psychological and social QoL scores were lower than the non-pandemic norms of the general population, while the physical health and environmental QoL scores were comparable. After adjusting for relevant demographic, personal, and clinical variables, religious coping; greater number of hours of online classes attended; and greater social support from family, friends and significant others were found to be significantly associated with higher QoL among the participants. Frustration because of study disruption, living in areas with a high prevalence of COVID-19 cases, and a higher severity of depressive and stress symptoms were significantly associated with lower QoL.

Conclusion: COVID-19 impaired the QoL of university students even after the movement lockdown was lifted.

\section{Introduction}

The severe acute respiratory syndrome coronavirus-2 (SARS-CoV-2) is a highly infectious and contagious virus belonging to the coronavirus family. Since its announcement by the World Health Organization (WHO) as a global pandemic on 11 March 2020, it has caused a major health hazard globally-the coronavirus disease 2019 (COVID-19) pandemic [1]. Malaysia, which has been experiencing an alarming increase in the prevalence of COVID-19 since early March 2020, imposed a movement control order (MCO) throughout the entire country from March 2020 to June 2020. Under the MCO, all forms of public gatherings for social, religious, sporting, or cultural purposes were banned, and all places of worship and business premises except for essential services were closed [2]. The MCO was lifted in June 2020 but the rate of spread of COVID-19 in the country was not fully under control. Fear of being infected with COVID19 and uncertainty about the future resulting from the socioeconomic downfall and academic disruption stemming from this global pandemic have enormous psychological effects on university students [3-7].

Quality of life (QoL) has emerged as an important measure in psychiatric research because of its frequent use as an assessment and treatment outcome indicator. The WHO's quality of life-BREF (WHOQoL-BREF) is a QoL measuring tool that can be used to compare health-related QoL across a huge 
variety of conditions or illnesses; it is also used as a tool to indicate the outcome of various QoL interventions [8]. Several factors, such as gender, education environment, years of study, depression, and chronic illness have been identified as predictors of QoL in university students [9]. In the Malaysian context, despite the MCO was lifted in June 2020, all academic activities were still confined, in which all classes are still conducted online since April 2020 and university students were not permitted to access the university's facilities. These new norms in the academic setting in Malaysia disrupt the usual daily routine and academic progress among university students. To the best of our knowledge, to date, data on QoL assessment in university students in response to the COVID-19 pandemic are lacking, particularly after the end of movement lockdown. Hence, this study filled the research gap via the following activities: (1) evaluating the QoL of university students and (2) assessing the association between various psychological factors, social support, and QoL to identify significant predictors of QoL among university students while adjusting for demographic, personal and clinical factors during the uncertain time of the COVID-19 pandemic and after the movement lockdown was lifted.

\section{Methods}

\section{Study setting and participants}

This cross-sectional online survey was conducted from 1 July 2020 to 21 July 2020, which was 3 weeks after the Malaysian government lifted the MCO (MCO was lifted on 11 June 2020). During the period of data collection, although the MCO had been lifted, the rate of spread of COVID-19 in the country was not fully under control, with the number of cumulative COVID-19 cases at 8840 cases and the number of deaths at 123 cases at the end of the data collection period [10]. The sample size was calculated based on the formula:

$n=\left[\left(Z_{1-a / 2} \times \mathbb{Z}\right) / \Delta\right]^{2}$ (where $n$ was the total estimated sample size, $Z_{1-a / 2}$ was the value represented the desired confidence interval in which confidence level selected was at $95 \%$ with a critical value of $1.96, \nabla$ was standard deviation which was 18.2 based on the QoL of the general population [11], and $\Delta$ was precision with a value of 2.5). Hence, the estimated sample size needed was 243 subjects (after considering an additional $20 \%$ of sample loss). Recruitment of study participants was carried out by snowball sampling from the medical faculties of Malaysian public university students in Klang Valley at the Central of Peninsular Malaysia and in the states of Penang and Kelantan located at the northern region of Peninsular Malaysia. Initially, the online survey was disseminated to medical postgraduate students and they were told to circulate the invitation to participate in the survey to other medical postgraduate students, medical undergraduate students, postgraduate and undergraduate students in medical sciences and other students within the medical faculties of public Malaysian universities located at the targeted regions. We selected participants with a diverse range of demographic characteristics according to age, gender and marital status. The study was approved by the Human Research Ethics Committee of USM (USM/JEPeM/COVID19-21) and the Medical Research Committee of the Faculty of Medicine, UKM (UKMPPI/111/8/JEP-2020-370). Those who were 18 years and above, registered as students with the Faculty of Medicine of Malaysian public universities located in Klang Valley and the 
states of Penang and Kelantan in Peninsular Malaysia, were eligible to participate in the study. Those who presented with psychotic disorders, bipolar mood disorder or a history of illicit drug use were excluded from the study. All the participants provided informed consent, and they were assured of anonymity and data confidentiality. They completed the questionnaires through an online survey platform (Google Forms). Initially, a total of 381 participants responded to the online survey. We excluded 65 participants who took less than $60 \%$ of the median time to complete the questionnaires in this study (median time $=15$ minutes) to avoid any response bias. Double responses from the same participant were prevented by activating the "limiting responses to once per person" function in Google Forms. The final sample size of the study was 316 participants.

\section{Data collection}

A self-report questionnaire was administered to the participants to collect data on the following: demographic and personal characteristics, clinical factors, and COVID-19 related stressors and coping of the participants. The self-reported questionnaire was constructed based on previous surveys on the psychological impact of the SARS and MERS epidemics on university and medical students [12-16]. The participants were also administered the Malay version of the 21 -item depression, anxiety and stress scale (DASS-21) to assess the severity of their depressive, anxiety and stress symptoms; the Malay version of the multidimensional scale of perceived social support (MSPSS) to assess the degree of social support; and the Malay version of the WHOQoL-BREF to assess QoL. In this study, the DASS-21 subscale scores, MSPSS domain scores and WHOQoL-BREF domain scores were presented as continuous variables.

\section{Demographic characteristics}

Data on demographic characteristics of the participants collected in this study included age, gender, marital status and monthly living expenses. The assessment and coding for demographic characteristics are summarized in Section 1 of the Supplementary material.

\section{Personal characteristics}

The personal characteristics assessed in this study were types of courses enrolled in university and living arrangement. The assessment and coding for personal characteristics are summarized in Section 1 of the Supplementary material.

\section{Clinical factors}

Data on two clinical factors were collected in this study, which were history of pre-existing medical illnesses and history of pre-existing depressive and anxiety disorders. The assessment and coding for clinical factors are summarized in Section 1 of the Supplementary material.

\section{COVID-19 related stressors and coping}


Data on COVID-19 related stressors and coping included in this study were hours of online classes attended per week, perceived prevalence of COVID-19 cases at place of living, frustration because of loss of daily routine, frustration because of disruption of study and use of religious coping to manage stress in response to the COVID-19 pandemic. The assessment and coding for COVID-19 related stressors and coping are summarized in Section 1 of the Supplementary material.

\section{Depression, anxiety and stress}

The presence of depression, anxiety and stress as well as the severity of these symptoms were evaluated with the DASS-21. The DASS-21 is a self-report questionnaire consisting of 21 items, with 7 items per subscale; the subscales are depression, anxiety and stress. Each item is scored on a Likert scale from 0 (did not apply to me at all) to 3 (applied to me very much). Sum scores are computed by adding the scores on the items per subscale and multiplying them by a factor of 2 . Sum scores for each of the subscales may range between 0 and 42 . Hence, the total score of the DASS-21 ranges from 0 to 120 . The cut-off scores for case findings in DASS-21 are as follows: 9 for the depression subscale, 7 for the anxiety subscale and 14 for the stress subscale [17]. The Malay version of the DASS-21 has good Cronbach's alpha values of $0.75,0.74$ and 0.79 for the depression, anxiety and stress subscales, respectively [18].

\section{Social support}

The perceived social support received from family, friends and significant others were measured by the MSPSS. The MSPSS is a self-administered instrument that measures the perceived adequacy of the available amount of social support individuals receive from friends, family and significant others/special persons. The MSPSS has 12 items, where each item is rated on a 7-point Likert scale ranging from 1 (very strongly disagree) to 7 (very strongly agree). Hence, the cumulative scores of the MSPSS range from 12 to 84. Each domain comprises four items; hence, the cumulative scores for each domain range from 4 to 28. The higher the score, the higher the level of perceived social support of the individual. The original version of the MSPSS has good internal consistency (Cronbach's $a=0.88$ ) [19]. The Malay version of the MSPSS has been validated among Malaysian university students, showing a high internal consistency (Cronbach's $\mathrm{a}=0.94)[20]$.

\section{Quality of life}

The quality of life of the participants was measured by the WHOQoL-BREF. The WHOQoL-BREF is a selfadministered questionnaire that was used to assess the QoL of the subjects. It comprises 26 items; items 1 and 2 are general questions on QoL, whereas the other items are grouped into four domains (i.e. physical health, psychological, social relationship and environment-related QoL. Each item is scored on a Likert scale ranging from 1 to 5 . Each domain is scored with values from 0 to 100 , with higher scores indicating better QoL. The WHOQoL-BREF has good psychometric properties [21]. The general norms for the WHOQoL-BREF domain scores are as follows: 70.6 (standard deviation $=14.0$ ) for psychological QoL, 73.5 (standard deviation $=18.1$ ) for physical health QoL, 75.1 (standard deviation $=13.0$ ) for environmental QoL and 71.5 (standard deviation = 18.2) for social relationships QoL [11]. The Malay 
version of the WHOQoL-BREF has also demonstrated excellent psychometric properties, with an internal consistency (Cronbach's a) of 0.89 [22].

\section{Statistical analysis}

Statistical analyses were performed with the Statistical Package for Social Sciences (SPSS) version 26 (SPSS 26; SPSS Inc., Chicago, Illinois, USA). Descriptive statistics were reported for demographic, personal, clinical factors and COVID-19 related stressors and coping of the participants, as well as for the DASS-21, MSPSS and WHOQoL-BREF domain scores (to achieve objective 1 of the study). All the categorical variables were presented as frequencies and percentages, while the continuous variables were presented as means and standard deviations. There were no missing data.

To achieve objective 2 of the study, simple and multiple linear regression analyses were used to examine the association between COVID-19 related stressors and coping, psychological factors, perceived social support and quality of life domains. In the multiple linear regression analyses, we adjusted relevant demographic, personal, and clinical variables. Multicollinearity was assessed by referring to the variance inflation factor, in which all the independent variables included in the multiple linear regression models had a score of $<5$, indicating no multicollinearity. The normal probability plot of residuals of all the multiple linear regression models demonstrated that all the points lay in a reasonably straight diagonal line from bottom left to top right, indicating that the errors of the linear regression models were normally distributed. Statistical significance was set at $p<0.05$ for the multiple linear regression analyses, and all $p$-values were two-sided.

\section{Results}

\section{Study participants}

All the participants completed all the questionnaires. The demographic, personal, clinical characteristics and COVID-19 related stressors and coping of the participants are summarised in Table 1.

The mean physical health QoL, psychological QoL, social relationship QoL and environment QoL scores were 75.31 (SD = 15.11), $67.72(S D=17.14), 68.32(S D=18.22)$ and 74.61 (SD = 13.68), respectively. The psychological characteristics, social support and QoL of the participants are presented in Table 2.

\section{Associations between various factors and physical health related QoL among the participants}

Table 3 illustrates the association between COVID-19 related stressors and coping, psychological characteristics, social support and physical health-related QoL among the participants. Simple linear regression revealed that several factors were significantly associated with physical health-related QoL, and these are listed in Table 3. However, the multiple linear regression model indicated that only three variables were significantly associated with higher physical health-related QoL, which were a greater number of hours of online classes attended per week $(B=0.291,95 \% \mathrm{Cl}=0.088$ to $0.494, p=0.005)$, higher family support ( $B=2.300,95 \% \mathrm{Cl}=0.856$ to $3.743, p=0.002)$ and higher friend support $(B=2.662$, 
$95 \% \mathrm{Cl}=1.219$ to $4.104, p<0.001)$. In contrast, presence of frustration because of study disruption $(\mathrm{B}=$ $-4.493,95 \% \mathrm{Cl}=-7.320$ to $-1.667, p=0.002)$, and greater severity of stress symptoms $(\mathrm{B}=-0.302,95 \% \mathrm{Cl}=$ -0.603 to $-0.001, p=0.049$ ) were significantly associated with lower physical health-related QoL. The multiple linear regression model contributed to a significant regression equation of $F(19,296)=16.793, p$ $<0.001$ with adjusted $R^{2}=0.488$.

\section{Association between various factors and psychological-related QoL among the participants}

Table 4 presents the association between COVID-19 related stressors and coping, psychological characteristics, social support, and psychological-related QoL among the participants. Simple linear regression illustrated that several factors were significantly associated with psychological-related QoL, and these are listed in Table 4. The multiple linear regression model indicated that higher family support $(\mathrm{B}=2.973,95 \% \mathrm{Cl}=1.631$ to $4.315, p<0.001)$, higher friend support $(\mathrm{B}=2.367,95 \% \mathrm{Cl}=1.027$ to $3.708, p$ $=0.001)$ and higher significant other support $(B=2.134,95 \% \mathrm{Cl}=1.007$ to $3.262, p<0.001)$ were significantly associated with higher psychological-related QoL. Only two variables were significantly associated with lower psychological-related QoL, which were the perception that the area of residence had a high prevalence of COVID-19 cases $(\mathrm{B}=-3.046,95 \% \mathrm{Cl}=-5.557$ to $-0.535, p=0.018)$ and greater severity of depressive symptoms $(B=-0.645,95 \% \mathrm{Cl}=-0.897$ to $-0.393, p<0.001)$. The multiple linear regression model contributed to a significant regression equation of $F(19,296)=32.616, p<0.001$ with adjusted $R^{2}=0.656$.

\section{Associations between various factors and social relationship QoL among the participants}

The associations between COVID-19 stressors and coping, psychological characteristics, social support, and social relationship QoL among the participants are summarised in Table 5. Simple linear regression indicated that several factors were significantly associated with social relationship QoL, and these are listed in Table 5. Nevertheless, the multiple linear regression model showed that only agreement that religious coping helped manage stress $(B=4.048,95 \% \mathrm{Cl}=0.798$ to $7.299, p=0.015)$, higher family support $(B=2.105,95 \% \mathrm{Cl}=0.383$ to $3.827, p=0.017)$, higher friend support $(B=5.307,95 \% \mathrm{Cl}=3.586$ to $7.028, p<0.001)$ and higher significant other support $(B=2.161,95 \% \mathrm{Cl}=0.714$ to $3.608, p=0.004)$ were significantly associated with higher social relationship QoL. None of the variables predicted lower social relationship QoL. The multiple linear regression model contributed to a significant regression equation of $F(19,296)=17.500, p<0.001$ with adjusted $R^{2}=0.499$.

\section{Associations between various factors and environment related QoL among the participants}

The association between COVID-19 related stressors and coping, psychological characteristics, social support, and environment QoL among the participants are illustrated in Table 6. Simple linear regression revealed that several factors were significantly associated with environment QoL, and these are listed in Table 6. The multiple linear regression model confirmed that agreeing that religious coping helped to manage stress $(B=3.947,95 \% \mathrm{Cl}=1.337$ to $6.558, p=0.003)$, higher family support $(\mathrm{B}=1.801,95 \% \mathrm{Cl}=$ 0.418 to $3.184, p=0.011)$, higher friend support $(B=3.101,95 \% \mathrm{Cl}=1.719$ to $4.483, p<0.001)$ and higher 
significant other support $(\mathrm{B}=2.367,95 \% \mathrm{Cl}=1.205$ to $3.529, p<0.001)$ were significantly associated with higher environment QoL. None of the variables predicted lower environmental QoL. The multiple linear regression model contributed to a significant regression equation of $F(19,296)=13.323, p<0.001$ with adjusted $R^{2}=0.426$.

\section{Discussion}

This study investigated the QoL of Malaysian university students and its association with various factors and social supports at a time when the country is still battling the COVID-19 pandemic and after the end of movement lockdown. As a comparison to the norms of the WHOQoL-BREF domain scores in the nonpandemic affected general population [11], the psychological (67.72 ${ }_{\text {[study] }}$ vs $70.6_{\text {[general population] }}$ ) and social relationship QoL levels (68.32 [study] vs $71.5_{\text {[general population] }}$ ) reported in our study were relatively low, whereas the physical health and environment QoL levels were comparable. This finding was not surprising because the prevalence rates of depression, anxiety and stress among the participants in this study were $36 \%, 37 \%$ and $42 \%$, respectively, which may lead to lower psychological QoL. Furthermore, the practice of social distancing and the restriction on organising and attending social activities as preventive measures to curb the spread of COVID-19 may contribute to lower social relationship QoL.

We found that only a greater number of hours of online classes attended per week and higher family and friend support significantly predicted an increase in physical health QoL among the participants. The literature pointed out that chronic absenteeism from class is associated with a higher risk of engaging in health risk behaviours, such as cigarette smoking, chronic alcohol use and risky sexual behaviours. In contrast, a sense of academic achievement is associated with a higher level of general health [23, 24]. Hence, the finding that university students who attended a greater number of hours of classes had higher physical health QoL in this study was in line with what was described in the literature. For the relationship between family and friend support and physical health QoL, a survey of 2348 adults in the United States reported that having good friend networking and friend support predicted increases in good subjective health status. Conversely, family and friend relationship strain may dampen long-term physical health [25]. In addition, greater family and friend support is related to increased moderate-and vigorous-intensity physical activity, which may enhance physical health-related QoL [26, 27]. Although our study did not assess the amount of physical activity engaged in by participants during the COVID-19 pandemic, increasing physical activities, such as exercise at home with family and friends, may be helpful to cope with boredom and a loss of daily routine, potentially enhancing the physical health QoL of the participants. Our findings identified that frustration because of study disruption and higher severity of stress symptoms significantly predicted a decrease in physical health QoL of the participants. Interestingly, further questioning of the participants indicated that they were complaining of uncertainty about their future as their study was prolonged, their graduation time would be delayed as a result of the COVID-19 pandemic and they were disturbed by loss of their daily academic routine, such as their usual classes and clinical sessions. These difficulties experienced by the participants were associated with increased severity of stress symptoms in this study. In fact, high level of stress among university 
students, particularly medical students may lead to stress-related physical exhaustion which may impaired their physical health-related QoL [28]. Hence, our study findings further strengthened the link between higher severity of anxiety symptoms and lower physical health QoL.

Four factors were identified as significant predictors of higher psychological QoL, which were as follows: higher levels of 1) family, 2) friend and 3) significant other social support. Conversely, higher severity of depression and perception of living in an area with high prevalence of COVID-19 cases significantly predicted lowering of psychological QoL. Studies on the general population and healthcare workers during the spread of the COVID-19 pandemic pinpointed that higher social support was associated with lower anxiety and depression, whereas lower social support was associated with higher anxiety and depression [29-33]. Greater family and friend support, greater integration into a social network and having a larger social network are also protective against depression [34]. Higher family and friend support have also been shown to enhance psychological well-being [35]. Hence, it is not surprising that higher family, friend and significant other social support for the participants in this study was associated with higher psychological QoL. Our finding that those who perceived the area in which they lived to have a high prevalence of COVID-19 cases showed reduced psychological QoL is similar to the findings of two studies in China, which also reported that those who live and work in close proximity to the epicentre of COVID-19 infection had higher odds of experiencing psychological symptoms, such as depressive and posttraumatic stress disorder symptoms $[33,36]$. The tighter movement control and fear of contracting the COVID-19 infection (for the self and family) in those who perceived that they lived in an area with a high prevalence of COVID-19 cases may have led to the emergence of higher negative affect, depreciating respondents' psychological QoL. Depression has been reported to diminish psychological QoL, and this is attributed to the mood disturbance experienced by the depressed person. The degree of decrement of psychological QoL is inversely proportional to the severity of depressive symptoms [37]. A study of 394 depressive disorder patients in Ethiopia reported that the psychological QoL domain of the WHOQoLBREF score were as low as $42.8 \pm 8.2$ [38]. Hence, our finding of the inverse relationship between depressive symptoms' severity and psychological QoL is well documented in the literature.

Our study indicated that using religious coping to manage their stress during the COVID-19 pandemic and having higher family, friend and significant other support predicted increased social relationship QoL among university students. No factors were significantly associated with lower social relationship QoL. Religious practices like attending religious services often increase the social network of attendees and allow frequent exchanges and sharing of information compared with attending such services less frequently [39]. It has been found that persons who attend religious services with one or both parents have greater promoted feelings of well-being, and those who attend religious services with their spouses exhibit enhanced relationship commitment [40]. Further questioning of the participants in our study revealed that those who attempted to cope with the MCO and COVID-19 pandemic with religious coping spent more time in prayers with family at home during the MCO; hence, they strengthened their family ties and enhanced their social relationship QoL further. These results may explain the reason behind our finding that those who utilised religious coping to manage stress reported better social relationship QoL. The COVID-19 pandemic has changed the quality of social relationships, where people receive more good 
support from their family, feel more caring towards family and others and share their feelings with others more often [41]. These shifts in social relationships support the association between higher family, friend and significant other support and greater social relationship QoL reported by the university students in this study.

The current study also highlighted that religious coping and greater family, friend and significant other support predicted an increase in the environmental QoL, while none of the COVID-19 related stressors and psychological complications were associated with lower environmental QoL among university students during the COVID-19 pandemic. Like our study, in which most participants were Muslim, Gardner et al. (2014) surveyed 114 Muslim university students in New Zealand and highlighted that religious coping was positively related with QoL [42]. Assessment of the individual domains of the WHOQoL-BREF also indicated that positive religious coping is associated with an increase in environmental QoL [43], supporting our finding that religious coping increased environmental QoL. Greater family, friend and significant other social support allow persons to strengthen their family ties, increase their social network size with friends and strengthen the positive relationship of a couple or partners. This may improve access of the person to resources and material goods, including financial support. Greater self-efficacy, competence and self-esteem as a result of good support from social networks may increase the sense of security of the physical surroundings and daily living, heightening environmental QoL [44]. Hence, it is not surprising that greater family, friend and significant other social support leads to higher environmental QoL, as reported by this study.

Based on the findings of this study, we can highlight a few recommendations to improve the QoL of university students during the COVID-19 pandemic. First, higher education institutions (HEls) should pay more attention to students who live in areas where COVID-19 cases are highly prevalent because these groups of students may have impaired QoL. Second, several psychological factors were reported to dampen QoL in this study, such as frustration because of study disruption and higher severity of depressive and anxiety symptoms. During the COVID-19 pandemic, when social distancing is pivotal as an infection preventive measure, online psychosocial interventions that help curb these psychological complications are of utmost importance. Hence, HEls should consider arranging online counselling or psychotherapy for university students needing these services. An example of an effective online psychosocial intervention for university students is the MePlusMe programme, which promotes psychological well-being, supports mood and daily functioning and enhances the study skills of university students [45]. Third, as religious coping and family, friend, and significant other social support increased the QoL of university students, HEls and the government may focus on efforts to organise more online social support groups, encourage the use of web-conferencing applications to sustain social communication and relationships and organise more online religious talks through HEl websites during the COVID-19 pandemic. Finally, a sufficient duration of online classes should be arranged to enhance the sense of academic satisfaction and reduce feelings of uncertainty among university students, considering that a greater number of hours of online classes attended improve the QoL of university students. 
There are a few limitations to take note of in this study. First, the cross-sectional design of this study did not allow the causal relationship between various factors and QoL to be determined across time. Second, as the participants were not sampled by random sampling, they may not be a representative sample of the university students in Malaysia. Despite these limitations, this study filled the research gap of the scarcity of data on QoL of university students after the movement lockdown ended and allowed several recommendations to be made.

\section{Conclusion}

In conclusion, this study indicated that university students had lower psychological and social relationship QoL levels in response to the COVID-19 pandemic even after the MCO was lifted. The current study identified two COVID related stressors which predicted lower QoL among university students: frustration because of study disruption and perception of living in an area with high prevalence of COVID19 cases. Two psychological factors were predictive of lower QoL: higher severity of depression and stress. Conversely, greater number of hours of classes attended per week, religious coping, higher family, friends and significant others social support were associated with higher QoL among university students. Our findings indicated the pivotal role of online mental healthcare services and social support groups, and we made some recommendations to improve the QoL of university students during the COVID-19 pandemic.

\section{Declarations}

\section{Funding}

This research was funded through grants to the corresponding author from Advanced Medical and Dental Institute, Universiti Sains Malaysia.

\section{Conflicts of interest}

All the authors declare no conflict of interest influencing the present work.

\section{Availability of data and material}

Data of the study available upon reasonable request.

\section{Code availability}

Not applicable

\section{Ethical approval}

The study was approved by the Human Research Ethics Committee of Universiti Sains Malaysia (USM/JEPeM/COVID19-21) and the Medical Research Committee of the Faculty of Medicine, Universiti 
Kabangsaan Malaysia (UKMPPI/111/8/JEP-2020-370).

\section{Consent to participate}

All participants consented to the study participation.

\section{Authors' contribution}

Bin Abdullah, M.F.I.L conceived and supervised the project and analysed the data. Mansor N.S. analysed the data and prepared the manuscript with support from Bin Abdullah, M.F.I.L. Teoh, S.H. and Mohammad, M.A. contributed in the design of survey questionnaire and data collection.

\section{References}

1. World Health Organization. WHO Director-General's opening remarks at the media briefing on COVID19 - 11 March 2020. https://www.who.int/dg/speeches/detail/who-director-general-s-openingremarks-at-the-media-briefing-on-covid-19-11-march-2020 (2020a). Accessed 19 Oct 2020.

2. Bunyan J. PM: Malaysia under movement control order from Wed until March 31, all shops closed except for essential services. The Malay Mail. 2020. https://www.malaymail.com/news/malaysia/2020/03/16/pm-malaysia-in-lockdown-from-wed-untilmarch-31-all-shops-closed-except-for/1847204. Accessed 19 Oct 2020.

3. Cao WJ, Fang ZW, Hou GQ, Han M, Xu, XR, Dong JX, et al. The psychological impact of the COVID-19 epidemic on college students in China. Psychiatry Res. 2020;287:112934.

4. Elmer T, Mepham K, Stadtfeld C. Students under lockdown: Comparisons of students' social networks and mental health before and during the COVID-19 crisis in Switzerland. PLoS ONE. 2020;15(7):e0236337.

5. Li YC, Wang Y, Jiang JW, Valdimarsdóttir UA, Fall K, Fang F, et al. Psychological distress among health professional students during the COVID-19 outbreak. Psychol Med. 2020; doi: $10.1017 /$ S0033291720001555.

6. Sundarasen S, Chinna K and Kamaludin K, Nurunnabi M, Mohammad Baloch G, Khoshaimet HB, al. Psychological impact of COVID-19 and lockdown among university students in Malaysia: implications and policy recommendations. Int J Environ Res Public Health. 2020;17(17):6206.

7. Zolotov Y, Reznik A, Bender S, Konstantinov V, Reznik A,1 Isralowitz R. (2020) COVID-19 fear, mental health, and substance use among Israeli university students. Int J Ment Health Addict. 2020; doi: 10.1007/s11469-020-00351-8.

8. Guyatt GH, Feeny DH, Patrick DL. Measuring health-related quality of life. Ann Intern Med. 1993;118(8):622-9.

9. Solis AC, Lotufo-Neto F. Predictors of quality of life in Brazilian medical students: a systematic review and meta-analysis. Braz J Psychiatry. 2019;41(6):556-67. 
10. World Health Organization. COVID-19 in Malaysia Situation Report 10. Available at: file:///C:/Users/User/Downloads/malaysia-situation-report-10.pdf (2020b). Accessed 23 Oct 2020.

11. Hawthorne G, Herrman H, Murphy B. Interpreting the WHOQOL-BRÈF: preliminary population norms and effect sizes. Soc Indic Res. 2006;77:37-59.

12. Wong JGWS, Cheung EPT, Cheung V, Cheung C, Chan MTY, Chua SE, et al. Psychological responses to the SARS outbreak in healthcare students in Hong Kong. Med Teach. 2004;26(7):657-66.

13. Loh L-C, Mohd Ali A, Ang T-H, Chelliah A. Impact of a spreading epidemic on medical students. Malays J Med Sci. 2006;13(2):30-6.

14. Wong TW, Gao Y, Tam WWS. Anxiety among university students during the SARS epidemic in Hong Kong. Stress Health. 2006;23(1):31-5.

15. Jeong H, Yim HW, Song YJ, Ki M, Min JA, Cho JH, et al. Mental health status of people isolated due to Middle East Respiratory Syndrome. Epidemiol Health. 2016;38:e2016048.

16. Preti E, Di Mattei V, Perego G, Ferrari F, Mazzetti M, Tarantoet, P., et al. The psychological impact of epidemic and pandemic outbreaks on healthcare workers: rapid review of the evidence. Curr Psychiatry Rep. 2020;22(8):43.

17. Lovibond SH, Lovibond PF. Manual for the Depression Anxiety Stress Scales. Sydney, NSW, Australia: Psychology Foundation of Australia; 1995.

18. Musa R, Fadzil MA, Zain Z. Translation, validation and psychometric properties of Bahasa Malaysia version of the Depression Anxiety and Stress Scales (DASS). ASEAN Journal of Psychiatry. 2007;8(2):82-9.

19. Zimet GD, Dahlem NW, Zimet SG, Farley GK. The multidimensional scale of perceived social support. J Pers Assess. 1988;52(1):30-41.

20. Ng CG, Amer Siddiq AN, Aida SA, Zainal NZ, Koh OH. Validation of the Malay version of the multidimensional scale of perceived social support (MSPSS-M) among a group of medical students in Faculty of Medicine, University Malaya. Asian J Psychiatr. 2010;3:3-6.

21. The WHOQOL Group. Development of the World Health Organization WHOQOL-BREF Quality of Life assessment. Psychol Med. 1998;28(3):551-8.

22. Hasanah $\mathrm{Cl}$, Naing L, Rahman ARA. World Health Organization Quality of Life Assessment: Brief Version in Bahasa Malaysia. Med J Malaysia. 2003;58(1):79-88.

23. El Ansari W, Stock $C$. Is the health and wellbeing of university students associated with their academic performance? cross sectional findings from the United Kingdom. Int J Environ Res Public Health. 2010;7(2):509-27.

24. Allison MA, Attisha E, Council On School Health. The link between school attendance and good health. Pediatrics. 2019;143(2):e20183648.

25. Walen HR, Lachman ME. Social support and strain from partner, family, and friends: Costs and benefits for men and women in adulthood. J Soc Pers Relat. 2000;17(1):5-30. 
26. Morrissey JL, Janz KF, Letuchy EM, Francis SL, Levy SM. The effect of family and friend support on physical activity through adolescence: a longitudinal study. Int J Behav Nutr Phys Act. 2015;12:103.

27. Smith GL, Banting L, Eime R, O'Sullivan G, van Uffelen JGZ. The association between social support and physical activity in older adults: a systematic review. Int J Behav Nutr Phys Act. 2017;14:56.

28. Ribeiro IJS, Pereira R, Freire IV, et al. Stress and quality of life among university students: a systematic literature review. Health Prof Educ 2018;4:70-7.

29. Cai WP, Lian B, Song XR, Hou TY, Deng GH, Li HF, et al. A cross-sectional study on mental health among health care workers during the outbreak of Corona Virus Disease 2019. Asian J Psychiatr. 2020;51:10211.

30. Li Y, Peng J. Coping strategies as predictors of anxiety: exploring positive experience of Chinese university in health education in COVID-19 pandemic. Creat Educ. 2020;11:735-50.

31. Liu Q, Luo D, Haase JE, Guo QH, Wang XQ, Liu S, et al. The experiences of health-care providers during the COVID-19 crisis in China: a qualitative study. Lancet Glob Health. 2020;8:e790-8.

32. Xiao H, Zhang Y, Kong DS, Li SY, Yang NX. The effects of social support on sleep quality of medical staff treating patients with Coronavirus Disease 2019 (COVID-19) in January and February 2020 in China. Med Sci Monit. 2020;26,e923549-1-e923549-8.

33. Song X, Fu W, Liu X, Luo ZQ, Wang RX, Zhou N, et al. Mental health status of medical staff in emergency departments during the Coronavirus disease 2019 epidemic in China. Brain Behav Immun. 2020;88:60-5.

34. Nguyen AW, Chatters LM, Taylor RJ, Mouzon DM. Social support from family and friends and subjective well-being of older African Americans. J Happiness Stud. 2016;17(3):959-79.

35. Mcllvane JM. Reinhardt JP. Interactive effect of support from family and friends in visually impaired elders. J Gerontol B Psychol Sci Soc Sci. 2001;56(6):374-82.

36. Guo J, Feng XL, Wang XH, van IJzendoorn MH. Coping with COVID-19: exposure to covid-19 and negative impact on livelihood predict elevated mental health problems in Chinese adults. Int $\mathrm{J}$ Environ Res Public Health. 2020;17(11):3857.

37. Berlim MT, Fleck MP. Quality of life and major depression. In: Ritsner MS and Awad AG. (eds) Quality of Life Impairment in Schizophrenia, Mood and Anxiety Disorders. Dordrecht: Springer; 2007. p. 241252.

38. Shumye S, Belayneh Z, Mengistu N. Health related quality of life and its correlates among people with depression attending outpatient department in Ethiopia: a cross sectional study. Health Qual Life Outcomes. 2019;17:169.

39. Brewer G, Robinson S, Sumra A, Tatsi E, Gire N. The influence of religious coping and religious social support on health behaviour, health status and health attitudes in a British Christian sample. J Relig Health. 2015;54(6):2225-34.

40. Science Daily. Religion or spirituality has positive impact on romantic/marital relationships, child development, research shows. 2014. https://www.sciencedaily.com/releases/2014/12/141208105320.htm. Accessed 18 Oct 2020. 
41. Zhang YF, Ma ZF. Impact of the COVID-19 pandemic on mental health and quality of life among local residents in Liaoning province, China: A cross-sectional study. Int J Environ Res Public Health. 2020;17(7):2381.

42. Gardner TM, Krägeloh CU, Henning MA. Religious coping, stress, and quality of life of Muslim university students in New Zealand. Ment Health Relig Cult. 2014;17(4):327-38.

43. Vitorino LM, Lucchetti G, Santos AEO, Lucchetti ALG, Ferreira EB, Adamiet NP, et al. Spiritual religious coping is associated with quality of life in institutionalized older adults. J Relig Health. 2016;55(2):549-59.

44. National Research Council (US) Panel on Understanding Divergent Trends in Longevity in HighIncome Countries. Explaining Divergent Levels of Longevity in High-Income Countries. Washington (DC): National Academies Press (US). https://www.ncbi.nlm.nih.gov/books/NBK62364/. 2011. Accessed 18 Oct 2020.

45. Barrable A, Papadatou-Pastou M, Tzotzoli P. Supporting mental health, wellbeing and study skills in higher education: an online intervention system. Int J Ment Health Syst. 2018;12:54.

\section{Tables}

Table 1. Demographic, personal, clinical characteristics, and COVID-19 related stressors and coping of the participants 


\begin{tabular}{|c|c|c|}
\hline Variables & $\mathbf{n}$ & $\%$ \\
\hline \multicolumn{3}{|l|}{ Demographic characteristics: } \\
\hline -Age: & $29.51^{\#}$ & $6.16^{\$}$ \\
\hline \multicolumn{3}{|l|}{ - Gender: } \\
\hline Male & 95 & 30 \\
\hline Female & 221 & 70 \\
\hline \multicolumn{3}{|l|}{-Marital status: } \\
\hline Married & 126 & 40 \\
\hline Single/divorcee/widowed & 190 & 60 \\
\hline \multicolumn{3}{|l|}{-Living expenses spent per month: } \\
\hline$\leq$ Ringgit Malaysia 3000 & 196 & 62 \\
\hline > Ringgit Malaysia 3000 & 120 & 38 \\
\hline \multicolumn{3}{|l|}{ Personal characteristics: } \\
\hline \multicolumn{3}{|l|}{-Types of course enrolled in university: } \\
\hline Medical science-based & 69 & 22 \\
\hline Medicine-based & 247 & 78 \\
\hline \multicolumn{3}{|l|}{-Living arrangement: } \\
\hline Live alone/with friends & 50 & 16 \\
\hline Live with family & 266 & 84 \\
\hline \multicolumn{3}{|l|}{ Clinical characteristics: } \\
\hline \multicolumn{3}{|l|}{-History of pre-existing medical illnesses: } \\
\hline No & 261 & 83 \\
\hline Yes & 55 & 17 \\
\hline \multicolumn{3}{|c|}{-History of pre-existing depressive and anxiety disorders: } \\
\hline \multicolumn{3}{|l|}{ No } \\
\hline Yes & 301 & 95 \\
\hline COVID-19 related stressors and coping: & 15 & 5 \\
\hline \multicolumn{3}{|l|}{-Frustration due to loss of daily routine: } \\
\hline \multicolumn{3}{|l|}{ No } \\
\hline Yes & 177 & 56 \\
\hline -Mean hours of online classes attended per week & 139 & 44 \\
\hline \multicolumn{3}{|l|}{-Frustration due to study disruption: } \\
\hline No & $5.49^{\#}$ & $3.45^{\$}$ \\
\hline
\end{tabular}


No

Yes

-Religion helped you to cope with stress during COVID-19?

Yes

${ }^{\#}=$ mean ${ }^{\$}=$ standard deviation

Table 2. Psychological characteristics, social support, and quality of life of the participants

\begin{tabular}{lcc}
\hline \multicolumn{1}{c}{ Variables } & Mean & Standard deviation \\
\hline Psychological characteristics: & & \\
-Mean DASS-21 Depression Subscale score & 8.53 & 8.37 \\
-Mean DASS-21 Anxiety Subscale score & 6.83 & 7.98 \\
-Mean DASS-21 Stress Subscale score & 10.52 & 8.95 \\
Social support: & & 4.87 \\
-Mean family support score & 22.28 & 4.72 \\
-Mean friend support score & 21.68 & 13.16 \\
-Mean significant other support score & 22.07 & 17.14 \\
Quality of life: & & 74.61 \\
-Mean physical health QoL score & 67.32 & \\
-Mean psychological QoL score & & \\
-Mean social QoL score & & \\
\hline
\end{tabular}

Table 3. The association between various factors and physical health-related QoL 


\begin{tabular}{cccc} 
& \multicolumn{2}{c}{ model $^{\mathrm{a}}$} \\
\hline B (95\% CI) & p- & B (95\% CI) & p-value \\
& value & &
\end{tabular}

COVID-19 related stressors and coping:

-Frustration due to loss of daily routine:

No

Yes

-Mean hours of online classes attended per week

\begin{tabular}{lcc} 
Reference & \multicolumn{2}{c}{ Reference } \\
& & \\
$.166(-12.384$ to & $<$ & $-2.455(-5.216$ to \\
$-5.949)$ & $0.001 *$ & $0.305)$
\end{tabular}

0.081

-Frustration due to study disruption:

No

Yes

$0.240(-0.014$ to $\quad 0.064 \quad 0.291(0.088$ to

positive cases?

0.493)

0.494)

No

Yes

-Religion helped you to cope with stress during COVID-19?

$\begin{array}{ccc}\text { Reference } & < & \text { Reference } \\ -8.367(-11.783 \text { to } & 0.001 * & -4.493(-7.320 \text { to } \\ -4.952) & & -1.667)\end{array}$

Psychological characteristics:

-Mean DASS-21 Depression Subscale score

0.131

-Mean DASS-21 Anxiety Subscale score

-Mean DASS-21 Stress Subscale score
Reference
$-3.647(-7.289$ to
$-0.005)$

0.050

Reference

Social support:

-Mean family support score

$-2.076(-4.778$ to

$0.625)$

0.162

-Mean friend support score

-Mean significant other support score

0.110

\begin{tabular}{|c|c|c|c|}
\hline Reference & & Reference & 0.653 \\
\hline $.910(-0.667$ to & & $1.942(-0.783$ to & \\
\hline 6.488$)$ & $<$ & $4.667)$ & 0.084 \\
\hline & .001 & & \\
\hline
\end{tabular}




\begin{tabular}{|c|c|c|c|}
\hline$-0.997(-1.164$ to & $<$ & $-0.062(-0.333$ to & $0.002 *$ \\
\hline \multirow[t]{2}{*}{$-0.830)$} & $0.001 *$ & $0.209)$ & $<$ \\
\hline & & & $0.001 *$ \\
\hline$-0.909(-1.093$ to & $<$ & $-0.251(-0.535$ to & \\
\hline-0.724$)$ & $0.001 *$ & $0.034)$ & 0.726 \\
\hline$-0.959(-1.113$ to & $<$ & $-0.302(-0.603$ to & \\
\hline \multirow[t]{2}{*}{$-0.804)$} & $0.001 *$ & $-0.001)$ & \\
\hline & $<$ & & \\
\hline 6.284 (5.068 to & $0.001 *$ & $2.300(0.856$ to & \\
\hline 7.499) & & 3.743) & \\
\hline 6.332 (5.102 to & $<$ & 2.662 (1.219 to & \\
\hline 7.561) & $0.001 *$ & 4.104) & \\
\hline 3.967 (2.836 to & & $0.216(-0.997$ to & \\
\hline 5.098) & & 1.429) & \\
\hline
\end{tabular}

$*$ = statistical significance at $\mathrm{p}<0.05 ; \mathrm{a}=$ multiple linear regression model reported that $F(19,296)=16.793, \mathrm{p}<0.001$ with adjusted $R^{2}=0.488$, adjusted for age, gender, marital status, living expenses, course enrolled in university, living arrangement, history of pre-existing medical, depressive and anxiety disorders

Table 4. The association between various factors and psychological-related QoL 


\begin{tabular}{cccc} 
& \multicolumn{3}{c}{ model $^{\mathrm{a}}$} \\
\hline B (95\% CI) & p- & B (95\% CI $)$ & p-value \\
& value & &
\end{tabular}

COVID-19 related stressors and coping:

-Frustration due to loss of daily routine:

No

Yes

-Mean hours of online classes attended per week

Reference

-Frustration due to study disruption:

$-9.321(-13.006$ to

$<$

Reference

0.082

No

$-5.637)$

$0.001 *$

$-2.277(-4.843$ to

Yes

$0.289)$

0.126

-Was your place of living highly prevalent for COVID-19

$0.202(-0.087$ to

0.170

positive cases?

$0.491)$

$0.147(-0.041$ to

$0.335)$

No

Yes

-Religion helped you to cope with stress during COVID-19?

No

Yes

Psychological characteristics:

-Mean DASS-21 Depression Subscale score

-Mean DASS-21 Anxiety Subscale score

-Mean DASS-21 Stress Subscale score

Social support:

-Mean family support score

-Mean friend support score

-Mean significant other support score

$-5.814(-9.776$ to

$-1.852)$

Reference

$0.004^{*}$

Reference

$-5.438(-9.550$ to

$-1.326)$

Reference

$0.370(-2.257$ to

2.998)

0.018*

$0.010 *$

$0 / 782$

\section{.}

\begin{tabular}{|c|c|c|}
\hline & Reference & \\
\hline & $-3.046(-5.557$ to & 0.061 \\
\hline $0.011^{*}$ & $-0.535)$ & \\
\hline
\end{tabular}

\section{Reference}

5.212 (1.180 to

$<$

Reference

$0.001^{*}$

9.245)

$0.001 * \quad 2.421(-0.112$ to
$4.954)$
$<$
$0.001 *$




\begin{tabular}{|c|c|c|c|}
\hline$-1.440(-1.601$ to & & $-0.645(-0.897$ to & \\
\hline-1.278$)$ & $<$ & $-0.393)$ & $<$ \\
\hline & $0.001 *$ & & $0.001 *$ \\
\hline$-1.119(-1.323$ to & & $-0.181(-0.446$ to & $0.001 *$ \\
\hline-0.916$)$ & $<$ & $0.083)$ & \\
\hline & $0.001 *$ & & $<$ \\
\hline$-1.204(-1.369$ to & $<$ & $-0.121(-0.401$ to & $0.001 *$ \\
\hline-1.038$)$ & $0.001 *$ & $0.159)$ & \\
\hline 9.082 (7.854 to & $<$ & 2.973 (1.631 to & \\
\hline 10.311) & $0.001 *$ & $4.315)$ & \\
\hline 8.500 (7.200 to & & 2.367 (1.027 to & \\
\hline 9.800$)$ & & 3.708) & \\
\hline 6.744 (5.589 to & & 2.134 (1.007 to & \\
\hline 7.899) & & $3.262)$ & \\
\hline
\end{tabular}

$*=$ statistical significance at $\mathrm{p}<0.05 ; \mathrm{a}=$ multiple linear regression model reported that $F(19,296)=32.616, \mathrm{p}<0.001$ with adjusted $R^{2}=0.656$, adjusted for age, gender, marital status, living expenses, course enrolled in university, living arrangement, history of pre-existing medical, depressive and anxiety disorders

Table 5. The association between various factors and social relationship-related QoL 
value

COVID-19 related stressors and coping:

-Frustration due to loss of daily routine:

No

Yes

-Mean hours of online classes attended per week

Reference

-Frustration due to study disruption:

$-7.319(-11.306$ to

$<$

Reference

0.762

No

$-3.332)$

$0.001 *$

$-0.508(-3.801$ to

Yes

2.785)

0.107

-Was your place of living highly prevalent for COVID-19

$0.235(-0.072$ to

0.133

positive cases?

$0.542)$

$0.199(-0.043$ to

$0.441)$

No

Yes

-Religion helped you to cope with stress during COVID-19?

Reference $\quad 0.004^{*}$

No

$-6.224(-10.435$ to

Reference

Yes

$-2.012)$

$-2.511(-5.882$ to

$0.861)$

Psychological characteristics:

-Mean DASS-21 Depression Subscale score

-Mean DASS-21 Anxiety Subscale score

0.185

-Mean DASS-21 Stress Subscale score

Reference

Social support:

$-2.973(-7.379$ to

1.433)

Reference

-Mean family support score

$-1.763(-4.985$ to

$0.015^{*}$

-Mean friend support score

$0.004^{*}$

1.459)

-Mean significant other support score

Reference

$6.353(2.080$ to

10.627)
$<$

0.001*

$<$

0.001*
0.491

Reference

4.048 (0.798 to

0.272

7.299)

0.144 


\begin{tabular}{|c|c|c|c|}
\hline$-1.068(-1.279$ to & & $-0.114(-0.437$ to & $0.017^{*}$ \\
\hline \multirow[t]{2}{*}{$-0.858)$} & $<$ & $0.210)$ & $<$ \\
\hline & $0.001 *$ & & $0.001 *$ \\
\hline$-0.861(-1.096$ to & & $-0.190(-0.529$ to & \\
\hline \multirow[t]{2}{*}{$-0.627)$} & $<$ & $0.150)$ & $0.004 *$ \\
\hline & $0.001 *$ & & \\
\hline$-0.913(-1.115$ to & $<$ & $-0.067(-0.426$ to & \\
\hline-0.711$)$ & $0.001 *$ & $0.292)$ & \\
\hline 8.547 (7.149 to & $<$ & 2.105 (0.383 to & \\
\hline 9.945) & $0.001 *$ & 3.827) & \\
\hline 9.576 (8.239 to & & 5.307 (3.586 to & \\
\hline 10.913) & & 7.028) & \\
\hline 6.895 (5.647 to & & 2.161 (0.714 to & \\
\hline 8.142) & & 3.608) & \\
\hline
\end{tabular}

$*$ = statistical significance at $\mathrm{p}<0.05 ; \mathrm{a}=$ multiple linear regression model reported that $F(19,296)=17.500, \mathrm{p}<0.001$ with adjusted $R^{2}=0.499$, adjusted for age, gender, marital status, living expenses, course enrolled in university, living arrangement, history of pre-existing medical, depressive and anxiety disorders

Table 6. The association between various factors and environmental-related QoL 


B $(95 \%$ CI $) \quad$ p- $\quad$ B $(95 \%$ CI $) \quad$ p-value

value

COVID-19 related stressors and coping:

-Frustration due to loss of daily routine:

No

Yes

-Mean hours of online classes attended per week

Reference

-Frustration due to study disruption:

$-4.879(-7.886$ to

$0.002^{*}$

Reference

0.299

No

$-1.873)$

Yes

$0.016^{*}$

$-1.399(-4.043$ to

-Was your place of living highly prevalent for COVID-19

$0.281(0.052$ to

positive cases?

$0.510)$

$0.186(-0.008$ to

$0.381)$

No

Yes

$0.007^{*}$

0.065

-Religion helped you to cope with stress during COVID-19?

Reference

No

$-4.390(-7.556$ to

Yes

$-1.223)$

Psychological characteristics:

-Mean DASS-21 Depression Subscale score

0.454

0.641

-Mean DASS-21 Anxiety Subscale score

-Mean DASS-21 Stress Subscale score

Reference

Social support:

$-1.263(-4.577$ to

Reference

-Mean family support score

2.051)

$0.008^{*}$

$0.614(-1.973$ to

0.003*

-Mean friend support score

3.202)

-Mean significant other support score

$\begin{array}{lccc}\text { Reference } & < & & 0.463 \\ 4.361(1.146 \text { to } & 0.001^{*} & \text { Reference } & \\ 7.576) & & 3.947(1.337 \text { to } & 0.062 \\ & < & 6.558) & \\ & & & \\ & & & \\ & & & \\ & & & \end{array}$




\begin{tabular}{|c|c|c|c|}
\hline$-0.690(-0.855$ to & $<$ & $-0.097(-0.357$ to & $0.011 *$ \\
\hline-0.526$)$ & $0.001 *$ & $0.163)$ & $<$ \\
\hline & & & $0.001 *$ \\
\hline$-0.544(-0.724$ to & $<$ & $-0.259(-0.532$ to & \\
\hline-0.363$)$ & $0.001 *$ & 0.013) & $<$ \\
\hline & $<$ & & $0.001 *$ \\
\hline$-0.588(-0.745$ to & $0.001 *$ & $0.051(-0.237$ to & \\
\hline-0.431$)$ & & $0.340)$ & \\
\hline & $<$ & & \\
\hline 5.658 (4.556 to & $0.001 *$ & 1.801 (0.418 to & \\
\hline 6.760$)$ & & 3.184) & \\
\hline 6.328 (5.255 to & & 3.101 (1.719 to & \\
\hline 7.400$)$ & & 4.483) & \\
\hline 4.756 (3.792 to & & 2.367 (1.205 to & \\
\hline 5.719) & & 3.529) & \\
\hline
\end{tabular}

$*$ = statistical significance at $\mathrm{p}<0.05 ; \mathrm{a}=$ multiple linear regression model reported that $F(19,296)=13.323, \mathrm{p}<0.001$ with adjusted $R^{2}=0.426$, adjusted for age, gender, marital status, living expenses, course enrolled in university, living arrangement, history of pre-existing medical, depressive and anxiety disorders

\section{Supplementary Files}

This is a list of supplementary files associated with this preprint. Click to download.

- SupplemetarymaterialstudentQoLResearchSquare.docx 